\title{
Lipid-Lowering Effects of Concurrent Training and Green Tea Consumption in Overweight Women
}

\author{
Zahra Hosseini', Hadi Ghaedi ${ }^{1 *}$, Mozhgan Ahmadi ${ }^{2}$, Seyed Ali Hosseini ${ }^{3}$ \\ ${ }^{1}$ Department of Sport Physiology, Lamerd Branch, Islamic Azad University, Lamerd; ${ }^{2}$ Department of Physical Education and Sport Science, Yadegar-e-Imam \\ Khomeini (RAH) Shahre-rey Branch, Islamic Azad University, Tehran; ${ }^{3}$ Department of Sport Physiology, Marvdasht Branch, Islamic Azad University, Marvdasht, Iran
}

Background: Exercise conducted simultaneously with the consumption of herbal supplements is one of the suggested methods for controlling obesity and its complications. The present study sought to investigate the lipid-lowering effects of concurrent training and green tea (GT) consumption in overweight women.

Methods: In this quasi-experimental study, 40 overweight women were selected and stratified into four groups: control (C), GT, concurrent training+placebo (TP), and concurrent training+GT (three tablets containing $500 \mathrm{mg}$ of GT per week; T+GT) groups. Both the TP and T+GT groups performed exercise three sessions per week for 8 weeks. Fasting blood samples were collected before and 48 hours after the last training session.

Results: Low-density lipoprotein cholesterol levels in the T+GT $(P=0.03)$ and TP $(P=0.001)$ groups were significantly decreased relative to in the GT group and were significantly decreased in the $T+G T$ group relative to in the TP group ( $P=0.001$ ). Meanwhile, high-density lipoprotein cholesterol levels in the T+GT group were significantly increased as compared with in the GT $(P=0.01), \operatorname{TP}(P=0.03)$, and C $(P=0.04)$ groups. Finally, total cholesterol levels in the T+GT group were decreased significantly as compared with in the TP, GT, and $C$ groups $(P=0.001)$ and triglyceride levels in the T+GT, TP, and GT groups were significantly increased as compared with in the $C$ group $(P=0.001)$.

Conclusion: Exercise performed simultaneously with GT consumption in comparison with either alone has a greater effect on improving the lipid profile in overweight women.

Key words: Exercise, Green tea, Overweight, Lipids

Received March 18, 2020

Reviewed April 13, 2020

Accepted November 1, 2020

${ }^{*}$ Corresponding author

Hadi Ghaedi

https://orcid.org/0000-0003-1497-7528

Department of Sport Physiology,

Lamerd Branch, Islamic Azad University, Lamerd 7434187456, Iran

Tel: +98-9177828667

Fax: +98-7152721232

E-mail: ghaedi.hadi@gmail.com

\section{INTRODUCTION}

Obesity is one of the most important health problems in the world and is associated with insulin resistance, changes in lipid metabolism, and metabolic syndrome. ${ }^{1}$ Levels of obesity, especially abdominal obesity, tend to be greater among women than men and women with high body mass index (BMI) and high lipid profile values are more likely to be infertile and experience polycystic syndrome, hyperlipidemia, and cancer. ${ }^{2}$ The lipid profile refers to the levels of different blood lipids (e.g., low-density lipoprotein cholesterol [LDL-C], high-density lipoprotein cholesterol [HDL-C], to- tal cholesterol [TC], and triglyceride [TG]) and the impairment of these factors increases the risk of heart disease. ${ }^{3}$ Increased physical activity and changes in dietary habits have been suggested as ways to reduce the complications of obesity. ${ }^{4}$ Studies have indicated that exercise is one of the key ways one can improve their lifestyle and its beneficial effects on cardiovascular disease-related factors have been well-demonstrated to date. ${ }^{5}$ Decreases in TC, LDL-C, and TG levels and increases in HDL-C levels after exercise have been observed $^{6,7}$ and are associated with health maintenance and disease prevention. Improvements in the lipid profile following aerobic ${ }^{8,9}$ and resistance ${ }^{10}$ training have been reported in various studies. 
However, some studies have identified concurrent training as an effective way to improve the lipid profile, body composition, and metabolic parameters in overweight and obese individuals. ${ }^{11}$

On the other hand, recent clinical and epidemiological studies have shown that rich bioactive compounds in plants known as phytochemicals can decrease the risk of cardiovascular disease and cerebrovascular events as well as certain types of cancer and oxidative stress. ${ }^{12}$ In this regard, green tea (GT) has received much attention due to its beneficial effects on health. GT contains caffeine; catechin; polyphenols; vitamins B, C, and E; flavonoids; glycoproteins; fiber; lipids; and carotenoids. ${ }^{13}$ Eating GT catechins reduces weight and lowers the risk of cardiovascular disease and diabetes. Also, by improving the serum lipid profile level, complications of metabolic syndrome can be avoided. ${ }^{14}$

Regarding the direct relationship between fats and myocardial infarction, the regulation of blood lipids is an important factor in health and, undoubtedly, the habit of proper physical activity plays an important role in this regard. On the other hand, the use of various medicinal plants has traditionally been widely adopted in the treatment of many ailments as well as in the improvement of sports performance. GT is one of the medicinal plants that, due to its high content of catechin, reduces body fat by increasing the metabolism. ${ }^{15}$ Given the above information as well as the few studies available on the interactive effects of exercise and GT consumption on the lipid profile in overweight people, the present study aimed to investigate the lipid-lowering effects of exercise combined with GT consumption in overweight women.

\section{METHODS}

\section{Study design}

We conducted an 8-week randomized controlled trial. This study was approved by the Research Ethics Committee of the Marvdasht Branch of Islamic Azad University (No. IR.IAU.M.REC.1398.627) on February 19, 2020. All participants provided written informed consent.

\section{Participants and the study enrollment process}

In this quasi-experimental study, 40 overweight women aged 27 to 38 years were selected as the statistical sample. Inclusion criteria were a BMI of 25 to $30 \mathrm{~kg} / \mathrm{m}^{2}$; no cardiovascular disease or metabolic disease (e.g., hypertension, diabetes, and dyslipidemia); no history of recurrent hypoglycemia during exercise; no regular exercise; no smoking; no adherence to a special diet; and no consumption of medications or supplements like statins, fibrates, omega-3 fatty acids, or steroids. Study participants were excluded from the final analysis of they withdrew from training, had more than three sessions of absenteeism, or became hospitalized. To match the 40 volunteers to specific groups, parameters such as age, height, weight, and BMI were measured. Also, general health and informed consent questionnaires were completed by all participants. Three days later, a 7-mL blood sample was collected from the right antecubital vein of each participant after 15 minutes of rest in the pretest period. Participants were then randomly divided into four groups of 10 people each, including the control (C), GT, concurrent training+ placebo (TP), and concurrent training $+\mathrm{GT}(\mathrm{T}+\mathrm{GT})$ groups. Before the study period, participants were asked to control their daily food intake by refusing to consume foods with high calorie and fat contents and to have their past diet. During 8 weeks, the GT and $\mathrm{T}+\mathrm{GT}$ groups consumed three tablets of $500 \mathrm{mg}$ GT per week, ${ }^{16}$ which consisted of polyphenols, catechin, epigallocatechin gallate, rice flour, magnesium stearate, capsule (vegetable cellulose). Also, the TP and T+GT groups performed exercise for three sessions per week. ${ }^{17}$ After 48 hours of washout time (for GT consumption) and the last training session or GT tablet consumption, similar to in the pretest period, all participants had $7 \mathrm{~mL}$ of blood collected from the right antecubital vein after 15 minutes of rest. An enzymatic method (calorimetry) with kits from Pars Azmon (Karaj, Iran) was used to measure direct levels of LDL-C, HDL-C, TG, and TC.

\section{GT consumption}

In this study, herbal tablets with a specified amount of catechin were used such that each tablet contained $500 \mathrm{mg}$ of GT containing $300 \mathrm{mg}$ catechin. The T+GT and GT groups consumed three tablets containing GT per week on odd days, while the TP group consumed three tablets containing a placebo (starch) on odd days. ${ }^{16}$

\section{Exercise protocol}

The exercise protocol was performed for 8 weeks and three ses- 
sions per week according to Table 1. Each exercise session consisted of three parts: a warm-up, the main part, and a cool-down. To warm up, the participants were directed to perform 10 minutes of walking, stretching, and slow running at $55 \%$ to $65 \%$ of their maximum heart rate (HRmax). Then, for the main portion of the exercise session, participants performed endurance and resistance training. Finally, during the cool-down, all participants completed walking and stretching training for 5 minutes at a level of intensity similar to that in the warm-up (Table 1) ${ }^{17}$

\section{Statistical analysis}

The Kolmogorov-Smirnov test was used to investigate the normal distribution of data, while a paired-samples t-test and one-way analysis of variance (ANOVA) with Tukey's post-hoc test were used to analyze the data in the IBM SPSS version 21 (IBM Corp., Armonk, NY, USA) $(P<0.05)$. The researchers received introduction letters from the Marvdasht Branch of Islamic Azad University with approval code IR.IAU.M.REC.1399.131.

Table 1. Exercise protocol

\begin{tabular}{|c|c|c|c|c|c|}
\hline \multicolumn{4}{|c|}{ Resistance training } & \multicolumn{2}{|c|}{ Endurance training } \\
\hline Week & Set & Repetition & Intensity & Time (min) & Intensity \\
\hline 1 & 2 & $16-18$ & 40\% 1RM & 16 & $61 \%$ HRmax \\
\hline 2 & 2 & $16-18$ & $45 \% 1 \mathrm{RM}$ & 16 & $66 \%$ HRmax \\
\hline 3 & 2 & $12-14$ & $50 \% 1 \mathrm{RM}$ & 20 & $70 \%$ HRmax \\
\hline 4 & 2 & $12-14$ & $55 \% 1 \mathrm{RM}$ & 20 & $74 \%$ HRmax \\
\hline 5 & 3 & $10-12$ & $60 \% 1 \mathrm{RM}$ & 25 & 77\% HRmax \\
\hline 6 & 3 & $10-12$ & $65 \% 1 \mathrm{RM}$ & 25 & $81 \%$ HRmax \\
\hline 7 & 3 & $8-10$ & $70 \% 1 \mathrm{RM}$ & 30 & $85 \%$ HRmax \\
\hline 8 & 3 & $8-10$ & $75 \% 1 \mathrm{RM}$ & 30 & $88 \%$ HRmax \\
\hline
\end{tabular}

RM, repetition maximum; HRmax, maximum heart rate.

Table 2. Demographic characteristics of the study participants

\begin{tabular}{lcccccc}
\hline \multirow{2}{*}{ Group } & \multirow{2}{*}{ Age $(\mathrm{yr})$} & \multicolumn{2}{c}{ Weight $(\mathrm{kg})$} & & \multicolumn{2}{c}{ BMl $\left(\mathrm{kg} / \mathrm{m}^{2}\right)$} \\
\cline { 3 - 4 } \cline { 6 - 7 } & & Pretest & Posttest & & Pretest & Posttest \\
\hline C & $32.12 \pm 3.83$ & $74.10 \pm 0.16$ & $75.22 \pm 1.11$ & & $29.00 \pm 3.68$ & $29.25 \pm 0.18$ \\
GT & $31.33 \pm 4.21$ & $74.20 \pm 5.08$ & $72.65 \pm 4.33$ & & $28.00 \pm 4.89$ & $27.81 \pm 5.08$ \\
TP & $32.18 \pm 2.12$ & $74.10 \pm 4.26$ & $73.10 \pm 2.87$ & & $28.10 \pm 2.76$ & $28.80 \pm 0.42$ \\
T+GT & $33.04 \pm 3.11$ & $73.00 \pm 6.96$ & $71.90 \pm 4.99$ & & $28.30 \pm 3.81$ & $28.21 \pm 4.22$ \\
\hline
\end{tabular}

Values are presented as mean \pm standard deviation. There was no difference in weight and BMl between pretest and posttest in C, GT, TP, and T+GT groups.

$\mathrm{BMI}$, body mass index; $\mathrm{C}$, control; $\mathrm{GT}$, green tea; TP, concurrent training+placebo; $\mathrm{T}+\mathrm{GT}$, concurrent training $+\mathrm{GT}$.

\section{RESULTS}

Demographic characteristics of the participants are presented in Table 2 and the lipid profile levels are reported in Figs. 1-4. The results of the Kolmogorov-Smirnov test indicated that the distribution of LDL-C $(P=0.67)$, HDL-C $(P=0.42)$, TC $(P=0.80)$, and TG $(P=0.61)$ in the pretest period and the distribution of LDL-C $(P=0.90)$, HDL-C $(P=0.51)$, TC $(P=0.58)$, and TG $(P=0.90)$ in the posttest period were normal.

The results of the paired-samples t-test showed that there were no significant changes between pretest and posttest values for both weight and $\mathrm{BMI}$ in the $\mathrm{C}, \mathrm{GT}, \mathrm{TP}$, and $\mathrm{T}+\mathrm{GT}$ groups $(P \geq 0.05)$. The results of the one-way ANOVA test showed that there were no significant differences in LDL-C $(P=0.12)$ or HDL-C $(P=0.88)$ levels in the pretest period among the four study groups; conversely, there were significant differences in the TC $(P=0.04)$ and TG

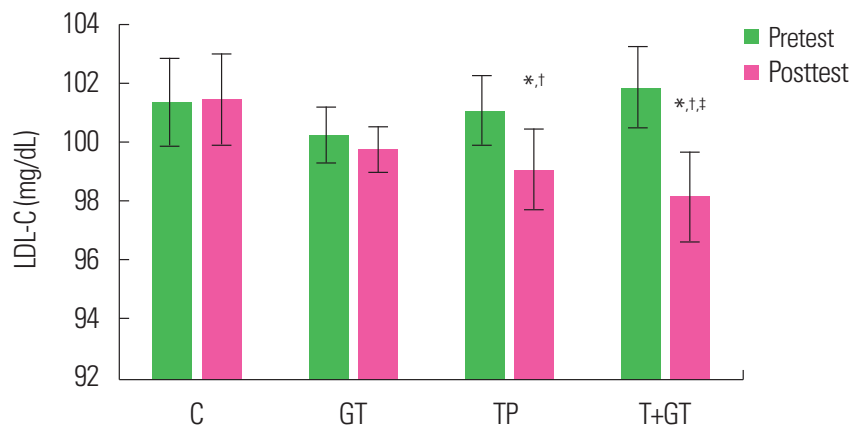

Figure 1. Serum low-density lipoprotein cholesterol (LDL-C) levels in the four research groups. *Significant decrease vs. pretest; 'Significant decrease vs. C group; 'Significant decrease vs. TP and GT groups. C, control; GT, green tea; TP, concurrent training+placebo; $\mathrm{T}+\mathrm{GT}$, concurrent training+GT.

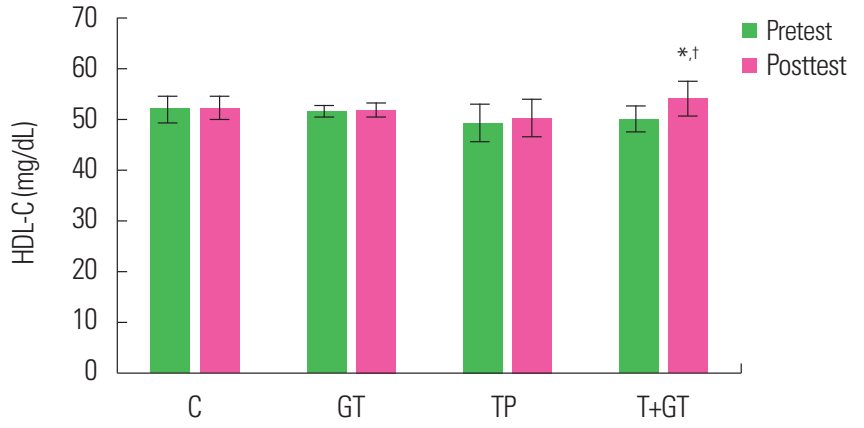

Figure 2. Serum high-density lipoprotein cholesterol (HDL-C) levels in the four research groups. *Significant increase vs. pretest; '`Significant increase vs. the TP, GT, and C groups. C, control; GT, green tea; TP, concurrent training+placebo; T+GT, concurrent training+GT. 


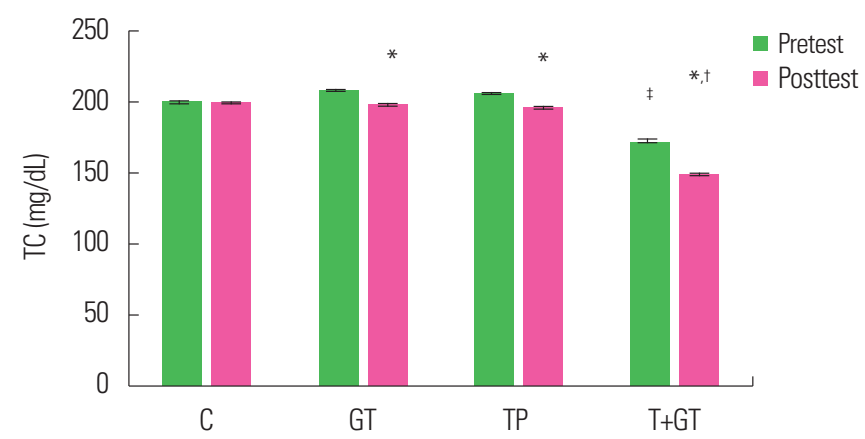

Figure 3. Serum total cholesterol (TC) levels in the four research groups. *Significant decrease vs. pretest; 'Significant decrease vs. TP, GT, and C groups; 'Significant decreased vs. GT group. C, control; GT, green tea; TP, concurrent training+ placebo; $\mathrm{T}+\mathrm{GT}$, concurrent training+GT.

$(P=0.03)$ levels. The results of Tukey's post-hoc test showed that TC levels in the $\mathrm{T}+\mathrm{GT}$ group were significantly lower than in the GT group $(P=0.02)$, while TG levels in $\mathrm{T}+\mathrm{GT}$ group were significantly lower than in the $\mathrm{C}(P=0.01)$, GT $(P=0.01)$, and TP groups $(P=0.01)$.

The results of the one-way ANOVA test showed that there were significant changes in LDL-C, HDL-C, TC, and TG levels in the four groups of the study $(P=0.001)$. The results of Tukey's post-hoc test showed that LDL-C levels in the T+GT $(P=0.03)$ and TP $(P=0.001)$ groups were significantly decreased as compared with in the GT group and those in the T+GT group were significantly decreased as compared with in the TP group $(P=0.001)$ (Fig. 1). Also, HDL-C levels in the $\mathrm{T}+\mathrm{GT}$ group were significantly relative to in the GT $(P=0.01)$, TP $(P=0.03)$, and $\mathrm{C}(P=0.04)$ groups (Fig. 2). TC levels in the T+GT group were significantly decreased as compared with in the TP, GT, and C groups $(P=0.001)$ (Fig. 3) and TG levels in the $\mathrm{T}+\mathrm{GT}$, TP, and GT groups were significantly decreased as compared with in the $\mathrm{C}$ group $(P=0.001)$ (Fig. 4).

The results of the paired-samples t-test showed that LDL-C levels in the T+GT $(P=0.001)$ and TP $(P=0.004)$ groups were significantly decreased posttest relative to in the pretest period; nevertheless, there were no significant changes between pretest and posttest levels in the GT $(P=0.10)$ and $\mathrm{C}(P=0.99)$ groups (Fig. 1). Meanwhile, HDL-C levels in the T+GT group posttest were significantly increased as compared with the pretest levels $(P=0.001)$, while there were no significant changes between the pretest and posttest levels in the GT $(P=0.69), \mathrm{TP}(P=0.61)$, and C $(P=0.34)$ groups (Fig. 2). The TC levels in the T+GT, TP, and GT groups in

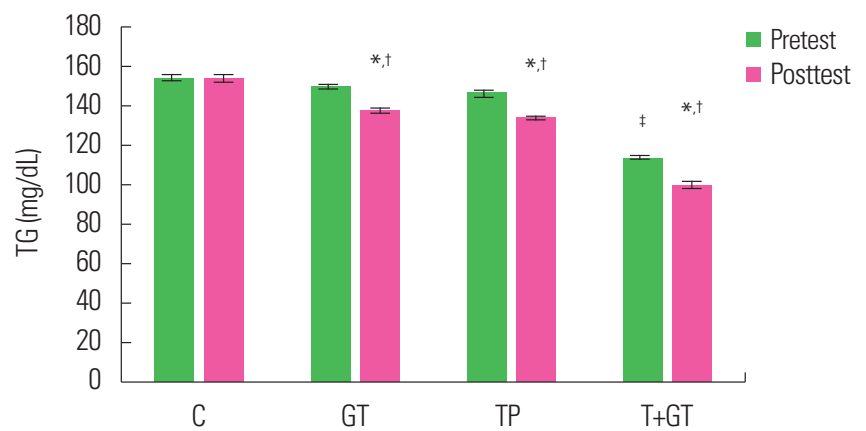

Figure 4. Serum triglyceride (TG) levels in the four research groups. *Significant decrease vs. pretest; 'Significant decrease vs. to $\mathrm{C}$ group; ${ }^{\ddagger}$ Significant decrease vs. C, GT, and TP groups. C, control; GT, green tea; TP, concurrent training+placebo; $\mathrm{T}+\mathrm{GT}$, concurrent training $+\mathrm{GT}$.

the posttest period were significantly decreased relative to in the pretest period $(P=0.001)$, while there were no such significant changes in the $\mathrm{C}$ group $(P=0.97)$ (Fig. 3). Finally, TG levels in T+GT, TP, and GT groups were significantly decreased posttest as compared with pretest $(P=0.001)$, yet there were no significant variations between pre- and posttest TG levels in the $\mathrm{C}$ group $(P=$ 0.79) (Fig. 4).

\section{DISCUSSION}

The results of the present study showed that 8 weeks of TP significantly decreased the serum levels of LDL-C, TG, and TC in overweight women. The results of this study are consistent with the findings of previous research. ${ }^{18,19}$ Since individuals' weight and changes in weight and body composition affect their lipoprotein profiles, ${ }^{20}$ significant changes in fat percentage, body weight, and body composition are likely to have a favorable effect on lipoprotein levels. In addition, the mechanism of the effect of exercise on improving the lipid profile is related to the enzymatic processes involved in lipid metabolism, where an increase in lipoprotein lipase (LPL) enzymatic activity has been reported. ${ }^{21,22}$ Changes in the lipid profile may also be correlated with other mechanisms, such as changes in plasma hormones and LPL concentrations. On the other hand, changes in blood lipids in response to exercise are dependent on early baseline levels of blood lipids. In fact, the main reason why exercises do not affect the blood lipids can be normal levels of HDL-C. ${ }^{23}$ Sugiura et al..$^{22}$ reported that regular exercise activity significantly decreased TC and LDL-C levels by increasing the activi- 
ty of LPL and lecithin-cholesterol acyltransferase. Other researchers have suggested that the change in LDL-C may be due to increased LPL and decreased hepatic lipase apolipoprotein B levels due to exercise. ${ }^{23}$

This study confirmed that 8 weeks of GT consumption significantly decreased TG and TC levels; however, there was no significant effect on HDL-C or LDL-C. The catechin in GT reduces lipid absorption and inhibits lipogenesis by reducing the effect on phospholipase A2. ${ }^{24}$ On the other hand, it seems that catechin in GT inhibits LDL-C oxidation by copper sulfate and inhibits cholesterol synthesis. Another possible mechanism of LDL-C depletion is related to the involvement of micelles of cholesterol in the digestive system, which, by forming insoluble cholesterol, can excrete cholesterol through the stool and reduce the degree of cholesterol absorption. On the other hand, polyphenols in GT increase HDL-C levels by inhibiting LDL-C oxidation and increasing serum antioxidant activity. ${ }^{25}$

Concerning the interactive effects, the results showed that exercise performed simultaneously with GT consumption significantly decreased the LDL-C, TG, and TC levels and increased the HDLC level; notably, our T+GT groups experienced a greater decrease in the LDL-C and TC levels and an increase in the HDL-C level as compared with the TP and GT groups. According to the findings of the present study, taking GT as an antioxidant supplement while performing regular exercise can help to reduce risk factors in overweight women. Contrary to the findings of the present study, however, some studies have shown that training with GT consumption has no interactive effect on improving the lipid profile in obese participants. ${ }^{26-28}$ However, differences in the results of this study and others may be attributed to are the sex of the study participants as well as variations between the compounds and the dose of GT. Moreover, these inconsistencies could be due to different training patterns. GT appears to be effective as a powerful antioxidant supplement in combination with exercise for controlling obesity and metabolic risk factors. Indeed, the catechins in GT reduce the absorption of lipids by inhibiting phospholipase A and inhibit lipogenesis by controlling the transcription of the fatty acid synthase and acetyl-coenzyme A carboxylase. ${ }^{18}$ Tracking the lipid level maintenance in the longer term after this study was one research limitation, so it is suggested that future investigations assess the lip- id maintenance two to 4 weeks after ceasing exercise to discern whether the beneficial effect persists. Although exercise and GT consumption alone improved the lipid profile in overweight women, however, exercise combined with GT consumption seems to have a more favorable effect on improving the lipid profile in this population.

\section{CONFLICTS OF INTEREST}

The authors declare no conflict of interest.

\section{ACKNOWLEDGMENTS}

The authors would like to thank all participants of this study.

\section{AUTHOR CONTRIBUTIONS}

Study concept and design: $\mathrm{ZH}$ and $\mathrm{HG}$; acquisition of data: $\mathrm{ZH}$; analysis and interpretation of data: $\mathrm{ZH}$; drafting of the manuscript: $\mathrm{MA}$ and SAH; critical revision of the manuscript: $\mathrm{MA}, \mathrm{SAH}$, and HG; statistical analysis: $\mathrm{ZH}$ and $\mathrm{SAH}$; obtained funding: $\mathrm{ZH}$; administrative, technical, or material support: $\mathrm{HG}, \mathrm{MA}$, and $\mathrm{SAH}$; and study supervision: HG.

\section{REFERENCES}

1. Bays HE, Toth PP, Kris-Etherton PM, Abate N, Aronne LJ, Brown WV, et al. Obesity, adiposity, and dyslipidemia: a consensus statement from the National Lipid Association. J Clin Lipidol 2013;7:304-83.

2. Pasdar Y, Darbandi M, Niazi P, Alghasi S, Roshanpour F. The prevalence and the affecting factors of obesity in women of Kermanshah. Jorjani Biomed J 2015;3:82-97.

3. Salehi Z, Salehi K, Moeini M, Kargarfard M, Sadeghi M. The effect of resistance exercise on lipid profile of coronary artery disease patients: a randomized clinical trial. Iran J Nurs Midwifery Res 2017;22:112-6.

4. American College of Cardiology/American Heart Association Task Force on Practice Guidelines, Obesity Expert Panel, 2013. Expert Panel Report: Guidelines (2013) for the man- 
agement of overweight and obesity in adults. Obesity (Silver Spring) 2014;22 Suppl 2:S41-410.

5. Zachariah G, Alex AG. Exercise for prevention of cardiovascular disease: evidence-based recommendations. J Clin Prev Cardiol 2017;6:109-14.

6. Skoumas J, Pitsavos C, Panagiotakos DB, Chrysohoou C, Zeimbekis A, Papaioannou I, et al. Physical activity, high density lipoprotein cholesterol and other lipids levels, in men and women from the ATTICA study. Lipids Health Dis 2003;2:3.

7. Trejo-Gutierrez JF, Fletcher G. Impact of exercise on blood lipids and lipoproteins. J Clin Lipidol 2007;1:175-81.

8. Wang Y, Xu D. Effects of aerobic exercise on lipids and lipoproteins. Lipids Health Dis 2017;16:132.

9. Kostrzewa-Nowak D, Nowak R, Jastrzębski Z, Zarębska A, Bichowska M, Drobnik-Kozakiewicz I, et al. Effect of 12-weeklong aerobic training programme on body composition, aerobic capacity, complete blood count and blood lipid profile among young women. Biochem Med (Zagreb) 2015;25: 103-13.

10. Costa RR, Lima Alberton C, Tagliari M, Martins Kruel LF. Effects of resistance training on the lipid profile in obese women. J Sports Med Phys Fitness 2011;51:169-77.

11. Shabani R, Jalali Z, Nazari M. Effects of concurrent strength and aerobic training on blood glucose homeostasis and lipid profile in females with overweight and obesity. Zahedan J Res Med Sci 2018;20:e13746.

12. Dembitsky VM, Poovarodom S, Leontowicz H, Leontowicz M, Vearasilp S, Trakhtenberg S, et al. The multiple nutrition properties of some exotic fruits: biological activity and active metabolites. Food Res Int 2011;44:1671-701.

13. Thielecke F, Boschmann M. The potential role of green tea catechins in the prevention of the metabolic syndrome: a review. Phytochemistry 2009;70:11-24.

14. Zheng XX, Xu YL, Li SH, Liu XX, Hui R, Huang XH. Green tea intake lowers fasting serum total and LDL cholesterol in adults: a meta-analysis of 14 randomized controlled trials. Am J Clin Nutr 2011;94:601-10.

15. Sugita M, Kapoor MP, Nishimura A, Okubo T. Influence of green tea catechins on oxidative stress metabolites at rest and during exercise in healthy humans. Nutrition 2016;32:321-31.
16. Amirhosein H, Elnaz E, Mohammadreza H. The effect of eight weeks aerobic training and moderate and high doses green tea consumption on body composition and lipid profile in overweight and obese women. Med J Mashhad Univ Med Sci 2015;58:359-69.

17. Asad M. Effect of 8 weeks aerobic, resistance and concurrent training on cholestrol, LDL, HDL and cardiovascular fitness in obesity male. Appl Res Sport Manag 2013;1:57-64.

18. Ghasemi E, Afzalpour ME, Zarban A. Effect of a 10 week high intensity interval training supplemented with green tea on lipid profiles and body composition in overweight women. J Birjand Univ Med Sci 2016;23:198-210.

19. Moradi H, Kolahdozi S, Ahmadi Kani Golzar F, Arabzade H, Asjodi F, Rezvan K. The Effects of eight weeks resistance training and green tea supplementation on cardiovascular risk factors in overweight men. J Shahrekord Univ Med Sci 2014; 16:77-87.

20. Kelley GA, Kelley KS. Impact of progressive resistance training on lipids and lipoproteins in adults: a meta-analysis of randomized controlled trials. Prev Med 2009;48:9-19.

21. Valle VS, Mello DB, Fortes Mde S, Dantas EH, Mattos MA. Effect of diet and indoor cycling on body composition and serum lipid. Arq Bras Cardiol 2010;95:173-8.

22. Sugiura H, Sugiura H, Kajima K, Mirbod SM, Iwata H, Matsuoka T. Effects of long-term moderate exercise and increase in number of daily steps on serum lipids in women: randomised controlled trial [ISRCTN21921919]. BMC Womens Health 2002;2:3.

23. Martins RA, Veríssimo MT, Coelho e Silva MJ, Cumming SP, Teixeira AM. Effects of aerobic and strength-based training on metabolic health indicators in older adults. Lipids Health Dis 2010;9:76.

24. Yang HY, Yang SC, Chao JC, Chen JR. Beneficial effects of catechin-rich green tea and inulin on the body composition of overweight adults. Br J Nutr 2012;107:749-54.

25. Esmaeil AM, Elham G, Asghar Z. Effects of an intensive resistant training sessions and green tea supplementation on malondialdehyde and total thiol in non-athlete women. Zahedan J Res Med Sci 2014;16:59-63.

26. Abedi B, Poorfahkimi Abarghu J, Ghadami A, Amini Rarani S. 
The effects of resistance training and green tea supplementation on lipid profile and insulin resistance in obese and overweight men. Complement Med J Fac Nurs Midwifery 2017; 7:1767-76.

27. Fathei M, Khairabadi S, Ramezani F, Hejazi K. Effect of eight weeks of aerobic training and green tea supplementation on cardiovascular risk factors in inactive overweight women. Horiz Med Sci 2016;22:283-9.

28. Javad V, Leila $H$. The effects of 8 weeks' aerobic exercise training along with green tea consumption on the cardiovascular risk factors in obese women. J Pract Stud Biosci Sport 2015; 3:78-88. 